\title{
A Brain Atlas of the Long Arm Octopus, Octopus minor
}

\author{
Seung-Hyun Jung ${ }^{\dagger}$, Ha Yeun Song ${ }^{\dagger}$, Young Se Hyun, Yu-Cheol Kim, \\ Ilson Whang, Tae-Young Choi and Seonmi Jo*
}

Department of Genetic Resources Research, National Marine Biodiversity Institute of Korea (MABIK), Seocheon 33662, Korea

\begin{abstract}
Cephalopods have the most advanced nervous systems and intelligent behavior among all invertebrates. Their brains provide comparative insights for understanding the molecular and functional origins of the human brain. Although brain maps that contain information on the organization of each subregion are necessary for a study on the brain, no whole brain atlas for adult cephalopods has been constructed to date. Here, we obtained sagittal and coronal sections covering the entire brain of adult Octopus minor (Sasaki), which belongs to the genus with the most species in the class Cephalopoda and is commercially available in East Asia throughout the year. Sections were stained using Hematoxylin and Eosin (H\&E) to visualize the cellular nuclei and subregions. H\&E images of the serial sections were obtained at 30 70- $\mu \mathrm{m}$ intervals for the sagittal plain and at 40 80- $\mu \mathrm{m}$ intervals for the coronal plain. Setting the midline point of the posterior end as the fiducial point, we also established the distance coordinates of each image. We found that the brain had the typical brain structure of the Octopodiformes. A number of subregions were discriminated by a Hematoxylinpositive layer, the thickness and neuronal distribution pattern of which varied markedly depending upon the region. We identified more than 70 sub-regions based on delineations of representative H\&E images. This is the first brain atlas, not only for an Octopodiformes species but also among adult cephalopods, and we anticipate that this atlas will provide a valuable resource for comparative neuroscience research.
\end{abstract}

Key words: Cephalopoda, Octopodiformes, Brain, Anatomy, Comparative histology

\section{INTRODUCTION}

With the exception of Nautilus, all extant cephalopods belong to the subclass Coleoidea, which can be divided into two superorders: Decapodiformes (10 arms) and Octopodiformes (8 arms) [1]. These cephalopods have the most complex nervous system of all

Received July 22, 2018, Revised August 5, 2018, Accepted August 9,2018

\footnotetext{
* To whom correspondence should be addressed. TEL: 82-41-950-0756, FAX: 82-41-750-0765 e-mail: joseonmi@mabik.re.kr

${ }^{\dagger}$ These authors contributed equally to this work.
}

invertebrates, and are characterized by large and specialized brain structures [2]. Although their brain structure is fundamentally different from that of vertebrates, these cephalopods show a number of sophisticated behaviors such as the use of tools [3], and thereby provide important comparative insights for understanding human brain and behavior. To investigate the molecular and network mechanisms underlying cephalopod brain function, the construction of a brain atlas that contains information on the organization of each brain region is an essential prerequisite. To date, however, no whole brain atlas of an adult cephalopod has been published. Although brain atlases have previously been constructed for the developmental and juvenile stages of certain Decapodiformes, such as the pygmy and oegopsid squids [4-7], none have been con- 
structed for the adult stage. Moreover, for Octopodiformes, which have the most centralized and cerebralized brain among cephalopods [8], no atlases have been constructed for any stage of the lifecycle. Although Young [9] has presented an extensive histological coverage of the brain of the common octopus, Octopus vulgaris Cuvier, it was not organized according to serial sections; hence, readers have difficulties in understanding the 3D structure of each sub-region. Therefore, it is necessary to construct the serial atlas of an adult cephalopod brain for further investigation, particularly that of Octopodiformes.

Within the Octopodiformes, the genus Octopus is notable for being the most speciose of the entire cephalopod class [8]. Among the species of Octopus, the long arm (whiparm) octopus, O. minor (Sasaki), is distributed throughout the coastal waters of Korea, China, Japan, and the Russian Sakhalin Islands [10]. The typical mantle and total lengths of $O$. minor are $10 \mathrm{~cm}$ and $70 \mathrm{~cm}$, respectively, and the total length at sexual maturity commonly reaches 50 $\mathrm{cm}$ [11]. Owing to its lower body weight and higher brain-to-body mass ratio when compared with $O$. vulgaris, which has been studied for a long time, $O$. minor is an economical model system for studying octopus brain and behavior. Furthermore, this species is a popular and commercially valuable component of fisheries in East Asia; therefore, it is relatively easy to obtain specimens throughout the year. In this study, we constructed a serial atlas of the brain of adult $O$. minor based on Hematoxylin and Eosin (H\&E) staining of serial sagittal and coronal sections throughout the entire brain. We also visualized the diversity of neuronal distribution patterns in each subregion by performing immunostaining with an antiacetylated $\alpha$-tubulin antibody and counterstaining with 4',6diamidino-2-phenylindole (DAPI).

\section{MATERIALS AND METHODS}

\section{Animals}

For the purposes of H\&E staining, we used two sexually mature female $O$. minor specimens, with a body weight of $160 \sim 180$ $\mathrm{g}$, obtained from commercial catches in the coastal regions of Jeollanam-do, South Korea, in May 2017. These specimens were identified as $O$. minor based on an examination of morphological characteristics [12]. Prior to experimentation, the two females were maintained for 3 months in filtered fresh seawater; during this time, they were fed with Manila clams. Comparison of the mitochondrial DNA (mtDNA) COI (cytochrome oxidase subunit I) barcode region [13] was performed to confirm species identity and determine haplotypes of the COI gene. Briefly, total DNA was isolated from the arm muscle of each animal using a DNeasy Blood \& Tissue kit (QIAGEN, USA), following the manufacturer's protocol. For amplification of the partial COI gene, polymerase chain reaction (PCR) was performed as previously described [14], using the primers HCO2198 and LCO1490 [15]. The PCR product of approximately $630 \mathrm{bp}$ was purified using a QIAquick PCR purification kit (QIAGEN, USA) and sequenced in an ABI 3730xl DNA Analyzer (Applied Biosystems, USA) using the same PCR primer set. The sequence data were edited and aligned using ATGC 4.0 software (Genetyx, Japan). For intact whole brain imaging and immunostaining, we used three sexually mature male O. minor specimens, with a body weight range of $220 \sim 290 \mathrm{~g}$, captured in June 2017 or June 2018, and identified based on morphological characteristics. For each animal, sex was determined by the presence of eggs in the mantle cavity (for females) or a hectocotylized right third arm (for males).

\section{Histology}

For H\&E staining, the animals were anesthetized by immersion in artificial seawater containing $2 \%$ ethanol for $8 \mathrm{~min}$. When they became relaxed and immobile, cartilaginous tissues containing the brain were excised and fixed with $10 \%$ formalin in artificial seawater at $4^{\circ} \mathrm{C}$ for 2 days. Paraffin-embedded serial sagittal or coronal sections at $10 \mu \mathrm{m}$ thickness were obtained from each brain (Histore Inc., Korea). Sections were stained with H\&E using a staining kit (ScyTek Laboratories, USA). Briefly, Mayer's Hematoxylin (Lillie’s modification) was applied to completely cover the tissue section and incubated for $5 \mathrm{~min}$. After rinsing twice with distilled water to remove any excess stain, a Bluing Reagent was applied for 10 15 s. Following two additional washes in distilled water, slides were dipped in absolute alcohol. Thereafter, the slides were incubated in adequate Eosin Y solution (modified alcoholic) for 2 3 min, rinsed, and dehydrated four times in absolute alcohol. The slides were then cleared and mounted with Histomount (National Diagnostics, USA).

\section{Image preparation}

Stereoscopic images of the fixed intact brain were taken after removing the cartilage surrounding the brain as completely as possible using forceps. Images of the intact brain and H\&E slide preparations were obtained under a LEICA S6D stereoscope (Leica Microsystems, Germany) using an OPTINITY KCS-2000SS CMOS camera (Korea Lab Tech, Korea). White balance was adjusted using OptiView 3.7 software (Korea Lab Tech, Korea). To establish the coordinates of each image, the distance from the midline point of the posterior end was calculated according to the thickness of the sections. Illustrations of brain gross anatomy were sketched by tracing a stereoscopic image of the intact brain using PowerPoint software (Microsoft, USA). For delineations, 40 
representative $H \& E$ images for each sagittal and coronal section were selected based on the appearance of distinguishable changes in structural morphology. The delineations were hand-drawn on a transparent film placed over each of the paper-printed H\&E images. Outlines of the brain were sketched with thick black lines, the brain internal structures with thin black lines, and Hematoxylin- stained layers with cyan lines or planes. All sketches were scanned and used as templates for tracing (Penodegr Inc., Korea) using Adobe Illustrator software (Adobe, USA).

\section{Nomenclature and abbreviations}

On the tracings, we indicated the name of each subregion with

A
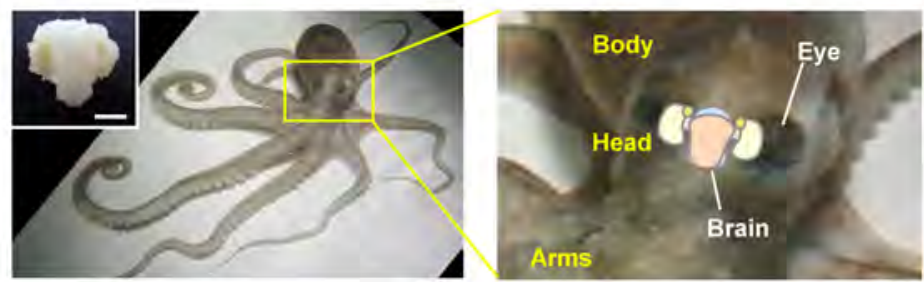

B
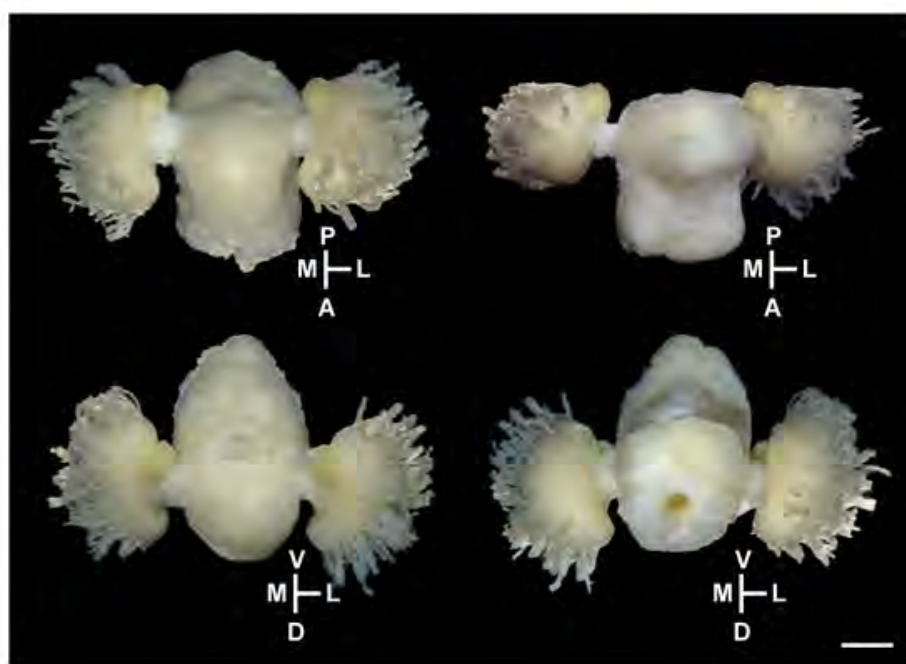

C

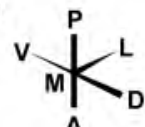

\section{Subesc}

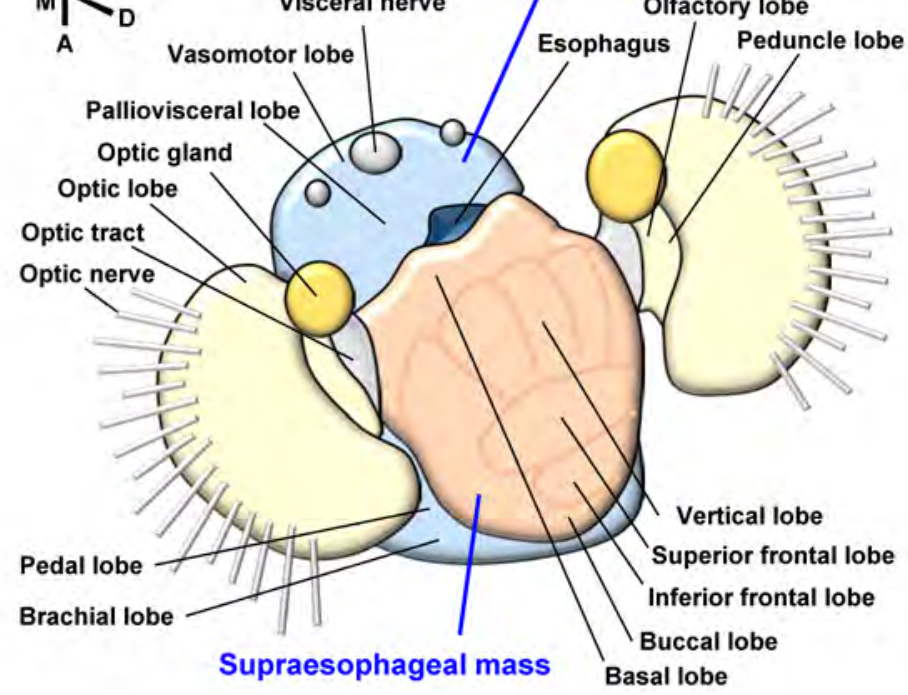

Fig. 1. The brain of Octopus minor. (A) Left: a live specimen of $O$. minor resting in seawater. Inset: an isolated $O$. minor brain with surrounding cranial cartilage. Scale bar $=5 \mathrm{~mm}$. Right: The yellow-lined box in the left panel is magnified to indicate the location of the brain. (B) Stereoscopic images of an $O$. minor brain from different views. Scale bar $=2 \mathrm{~mm}$. (C) Gross anatomy of the $O$. minor brain. A, anterior; P, posterior; $\mathrm{D}$, dorsal; $\mathrm{V}$, ventral; $\mathrm{M}$, medial; $\mathrm{L}$, lateral. 
abbreviations, using Adobe Illustrator software (Adobe, USA). For efficient conveyance of information among cephalopod researchers, we adopted the terms used in previous studies. All nomenclatures and abbreviations used are based on those presented in the book "The anatomy of the nervous system of Octopus vulgaris" [9]. With reference to the subregions identified on non-serial, partial images of $O$. vulgaris brain sections, subregions of the $O$. minor brain were carefully identified by the appearance and disappearance of distinct structures along the serial H\&E images. The organization of this atlas is based upon that of the Paxinos mouse brain atlas [16].

\section{Immunostaining}

For immunofluorescence staining in the supraesophageal and subesophageal masses, $10-\mu \mathrm{m}$-thick sagittal paraffin sections were prepared using the same procedure as described for H\&E staining. In case of the optic lobe, $30-\mu \mathrm{m}$-thick free-floating sagittal sections were prepared using a VT-1200S vibratome (Leica, Germany), after fixation with $4 \%$ paraformaldehyde overnight at $4^{\circ} \mathrm{C}$, followed by dehydration and rehydration. Immunofluorescence staining of neurons was carried out as previously described using an anti-acetylated $\alpha$-tubulin antibody (1:500; Sigma-Aldrich, USA) [17]. For fluorescent detection of antibody labeling, we used an Alexa Fluor 568 goat anti-mouse conjugate (1:1000; Molecular Probes, USA). DAPI (ThermoFisher, USA) counterstaining was performed for visualizing cell nuclei. Fluorescence images were obtained using a BX53 digital fluorescence microscope (Olympus, Japan).

\section{RESULTS AND DISCUSSION}

\section{The gross anatomy of the $O$. minor brain}

We initially isolated the brain with surrounding cranial cartilage, which is located inside the head of O. minor (Fig. 1A). After complete removal of cartilage, the intact $O$. minor brain (Fig. 1B) showed a typical brain morphology of the Octopodiformes brain [8]. The gross anatomy of the brain indicated that the supraesophageal and subesophageal masses are distributed around the esophagus, and the optic and olfactory lobes are bilaterally attached (Fig. 1C). Their orientation was determined by the position adopted by the animal during normal horizontal locomotion, as reported in previous studies [8]. In detail, along the esophagus, the direction toward the mouth is anterior, and on the opposite side, it is posterior. The direction towards the midline, which divides the brain symmetrically, is medial, whereas the direction away from the midline is lateral. The supraesophageal mass is considered to be the dorsal part, and the subesophageal mass is the ventral part.

\section{Construction of the serial brain atlas of adult $O$. minor}

Prior to preparing tissue for construction of the $O$. minor brain atlas, we confirmed the species identity of the animals by comparing nucleotide sequences of the mtDNA COI gene, which is the most commonly used DNA barcode region for invertebrates, including $O$. minor [13]. Sequence identification of the mtDNA COI gene through NCBI GenBank database (http://www.ncbi. nlm.nih.gov/) revealed that the sequences of the two specimens used for this atlas (sagittal section and coronal section, respectively) showed over $99 \%$ sequence identity to that of $O$. minor (GenBank accession No. HQ638215.1), 87\% similarity to $O$. kaurna (AY545188.1), and 85\% similarity to O. hubbsorum (AB385874). Within the 630-bp region of the COI gene, 12 variable sites were recognized (Fig. 2A) as previously reported [14]. They exhibited a haplotype 1 pattern that is found in Korean populations, with a high haplotype frequency distribution value (0.7 1.0), compared with haplotype 6 , which is found only in three populations in China (Fig. 2A).

We subsequently obtained serial sagittal sections covering the entire brain of the sequence-confirmed, sexually mature adult O. minor (Fig. 2B). To establish the coordinates of each section in this atlas, we selected the midline point of the posterior end as the fiducial point (asterisk in Fig. 2B). Sections were stained with $\mathrm{H} \& \mathrm{E}$ to visualize the cellular nuclei and to facilitate subregion discrimination (Fig. 2C). We obtained 122 sagittal images of the serial sections at 30 70- $\mu \mathrm{m}$ intervals (Supplementary Materials 1). Among the images, we carefully selected 40 images that sufficiently represented the complex structure of the $O$. minor brain. After preparing delineated illustrations of the selected images (Fig. $2 \mathrm{D}$ ), we established the name of each subregion and denoted it accordingly (Supplementary Materials 2). We also prepared serial coronal sections covering the entire brain of the other sequenceconfirmed sexually mature adult $O$. minor (Fig. 2E). As with the sagittal sections, coronal sections were stained using H\&E (Fig. $2 \mathrm{~F})$. We obtained 112 coronal images of the serial sections at 40 80- $\mu \mathrm{m}$ intervals (Supplementary Materials 3). Forty representative images were selected for illustration (Fig. 2G) and subregion identification (Supplementary Materials 4). On the basis of delineation of representative H\&E images, we identified more than 70 subregions, the abbreviations of which are listed in Table 1. We followed the nomenclatures and abbreviations used in Young's book [9], because it describes the largest number of subregions among the previous cephalopod studies and $O$. vulgaris belongs to the same genus with $O$. minor.

In the constructed atlas, the $O$. minor brain showed many compartmentalized structures which might be involved in particular brain functions such as motor control and cognition. A number 


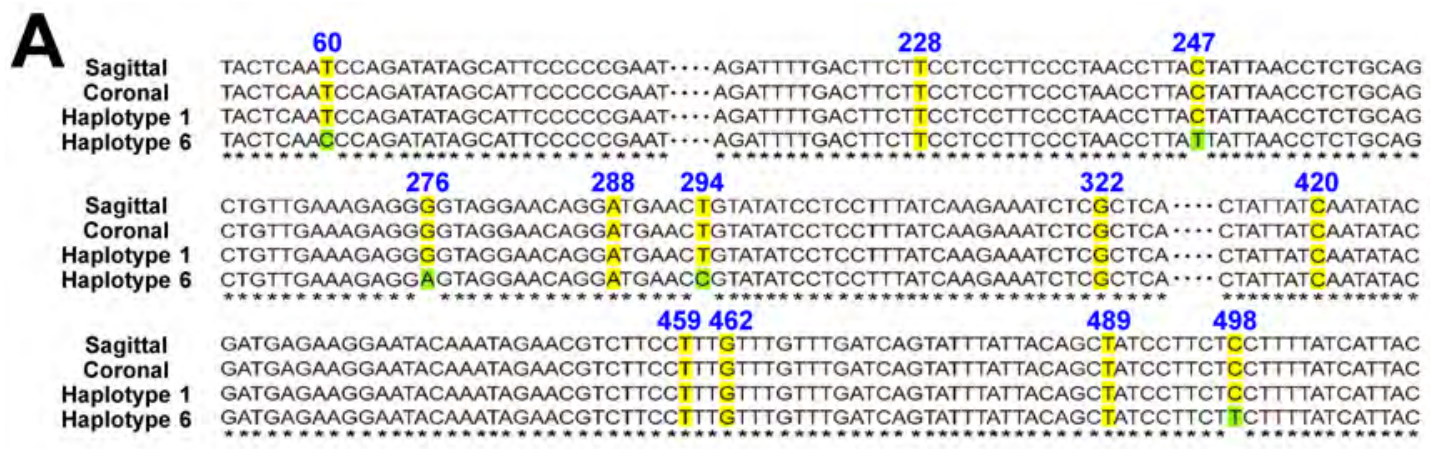

B

Sagittal section

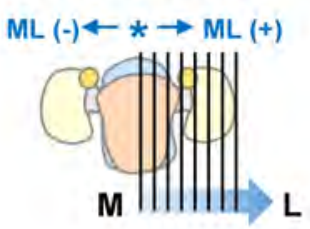

C

\section{$\mathrm{ML}+0.36 \mathrm{~mm}$}

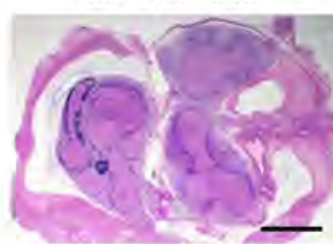

$\mathrm{ML}+2.28 \mathrm{~mm}$

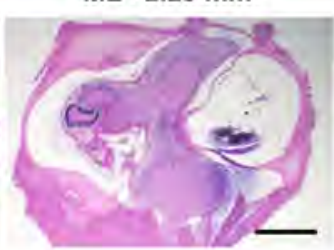

$\mathrm{ML}+4.68 \mathrm{~mm}$

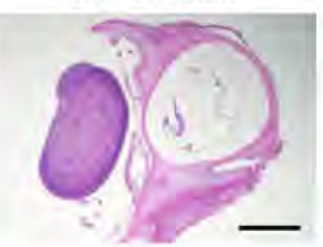

D

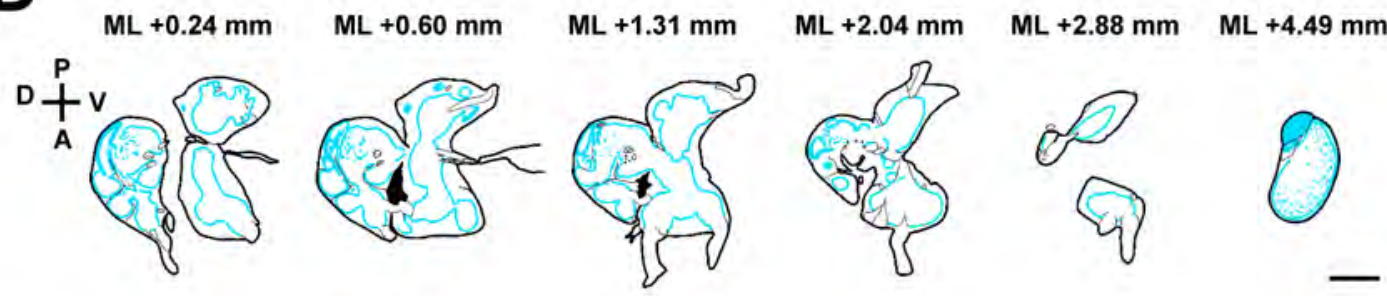

E

Coronal section

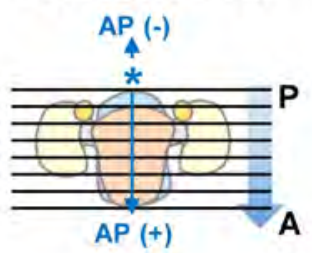

$\mathbf{F}$

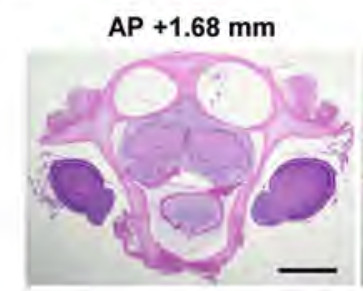

$A P+2.88 \mathrm{~mm}$

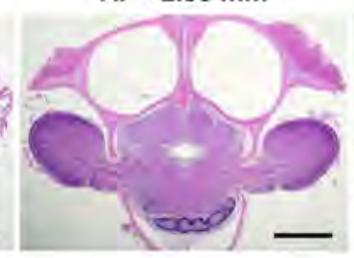

AP $+4.15 \mathrm{~mm}$

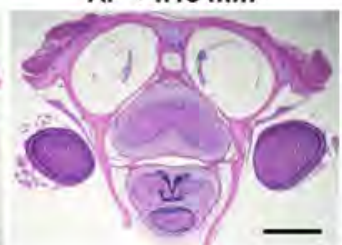

G

$A P+0.73 \mathrm{~mm} \quad A P+1.44 \mathrm{~mm}$

$A P+2.76 \mathrm{~mm}$

$\mathrm{AP}+3.69 \mathrm{~mm}$

$A P+4.21 \mathrm{~mm} \quad A P+5.24 \mathrm{~mm}$
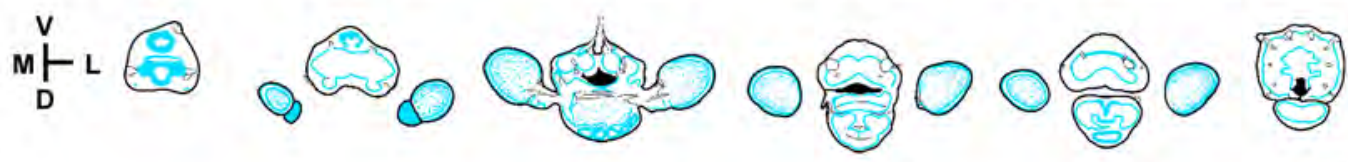

Fig. 2. Construction of a brain atlas of adult Octopus minor. (A) Genotyping of the animals used in atlas construction. ClustalW multiple sequence alignment of the mitochondrial cytochrome oxidase subunit I gene with known sequences of Haplotype 1 and Haplotype 6 [14]. The 12 nucleotide polymorphisms from Kang's study [14] are depicted as colored boxes, with the nucleotide number. Asterisks indicate nucleotides identical to those in Haplotype 1. (B) Schematic diagram of the sagittal section and established coordinates of the brain. (C) Representative images of the sagittal sections visualized by Hematoxylin and Eosin staining, shown with their coordinates. Scale bar $=2 \mathrm{~mm}$. (D) Representative images of delineations with their coordinates drawn on a selected sagittal section. Scale bar $=2 \mathrm{~mm}$. (E) Schematic diagram of the coronal section and established coordinates of the brain. (F) Representative images of the coronal sections visualized by Hematoxylin and Eosin staining, shown with their coordinates. Scale bar=2 mm. $(\mathrm{G})$ Representative images of delineations with their coordinates drawn on a selected coronal section. Scale bar=2 mm. AP, anterior-posterior axis; ML, mediallateral axis; \pm , coordinate direction; Asterisk, fiducial point; A, anterior; P, posterior; D, dorsal; V, ventral; M, medial; L, lateral. 
Table 1. List of structures identified in the brain of Octopus minor and the respective abbreviations

\begin{tabular}{|c|c|}
\hline Abbreviation & Structure \\
\hline b.a. & Anterior basal lobe \\
\hline b.d.a. & Anterior dorsal basal lobe \\
\hline b.d.p. & Posterior dorsal basal lobe \\
\hline b.int. & Interbasal lobe \\
\hline b.l. & Lateral basal lobe \\
\hline b.med. & Median basal lobe \\
\hline b.sul. & Basal sulcus \\
\hline br. & Brachial lobe \\
\hline br.po. & Postbrachial lobe \\
\hline br.pr. & Prebrachial lobe \\
\hline br.-pv.con. & Brachio-palliovisceral connective \\
\hline buc.p. & Posterior buccal lobe \\
\hline buc.s. & Superior buccal lobe \\
\hline c.br.sup. & Suprabrachial commissure \\
\hline c.opt.ven. & Ventral optic commissure \\
\hline cer-br.con. & Cerebro-brachial connective \\
\hline cer.con.p. & Posterior cerebral connective \\
\hline ch.p. & Posterior chromatophore lobe \\
\hline fri.l. & Lateral inferior frontal lobe \\
\hline fri.med. & Median inferior frontal lobe \\
\hline fr.s.l. & Lateral superior frontal lobe \\
\hline fr.s.med. & Median superior frontal lobe \\
\hline fun.p. & Posterior funnel lobe \\
\hline g.opt. & Optic gland \\
\hline mag.d. & Dorsal magnocellular lobe \\
\hline mag.p. & Posterior magnocellular lobe \\
\hline n.antorb.s. & Superior antorbital nerve \\
\hline n.antorb.i. & Inferior antorbital nerve \\
\hline n.br.1,l.r. & Lateral root of the first brachial nerve \\
\hline n.br.1,med.r. & Medial root of the first brachial nerve \\
\hline n.br.1. & First brachial nerve \\
\hline n.br.2,l.r. & Lateral root of the second brachial nerve \\
\hline n.br.2,med.r. & Medial root of the second brachial nerve \\
\hline n.br.2. & Second brachial nerve \\
\hline n.br.3,l.r. & Lateral root of the third brachial nerve \\
\hline n.br.3,med.r. & Medial root of the third brachial nerve \\
\hline n.br.3. & Third brachial nerve \\
\hline n.br.4,l.r. & Lateral root of the fourth brachial nerve \\
\hline n.br.4,med.r. & Medial root of the fourth brachial nerve \\
\hline n.br.4. & Fourth brachial nerve \\
\hline n.col. & Collar nerve \\
\hline n.fun.a. & Anterior funnel nerve \\
\hline n.fun.p. & Posterior funnel nerve \\
\hline n.lab. & Labial nerve \\
\hline n.pal. & Pallial nerve \\
\hline n.sal.p. & Posterior salivary nerve \\
\hline n.st. & Static nerve \\
\hline n.vc.a. & Anterior vena cava nerve \\
\hline n.visc. & Visceral nerve \\
\hline ol. & Olfactory lobe \\
\hline opt. & Optic lobe \\
\hline pe.a. & Anterior pedal lobe \\
\hline pe.p. & Posterior pedal lobe \\
\hline ped. & Peduncle lobe \\
\hline ped.sub. & Subpedunculate lobe \\
\hline prec. & Precommissural lobe \\
\hline
\end{tabular}

Table 1. Continued

\begin{tabular}{ll}
\hline Abbreviation & \multicolumn{1}{c}{ Structure } \\
\hline pv. & Palliovisceral lobe \\
subfr. & Subfrontal lobe \\
subv.a. & Anterior subvertical lobe \\
subv.p. & Posterior subvertical lobe \\
tr.br.-opt. & Brachial-optic lobe tract \\
tr.br.-pv. & Brachial-palliovisceral lobe tract \\
tr.cer. & Cerebral tract \\
tr.opt. & Optic tract \\
v. & Vertical lobe \\
v.l. & Lateral vertical lobe gyrus \\
v.med. & Median vertical lobe gyrus \\
v.med.l. & Medio-lateral vertical lobe gyrus \\
vas.d.l. & Lateral dorsal vasomotor lobe \\
vas.d.med. & Median dorsal vasomotor lobe \\
vas.ven. & Ventral vasomotor lobe \\
\hline
\end{tabular}

of lobes were discriminated by the Hematoxylin-positive layer (Supplementary Materials 1 and 3). We observed that the subesophageal mass consisted of brachial, pedal, chromatophore, funnel, vasomotor, and other lobes (Fig. 3A, 3B, and Supplementary Materials 2 and 4). These are known to be lower and intermediate motor centers. According to previous studies that performed electrical stimulations and induced lesions in the brains of $O$. vulgaris, the anterior part of the subesophageal mass is involved in the actions of arms and suckers, and the posterior part is involved in the actions of the mantle and viscera $[8,9]$. Compared with the subesophageal mass, the supraesophageal mass showed a more complex structure, consisting of multiple lobes, including the vertical, basal, frontal, and buccal lobes (Fig. 3A, 3B, and Supplementary Materials 2 and 4). In O. vulgaris, the basal lobes are known to be higher motor centers that are responsible for fine motor control and coordination, and other supraesophageal lobes are implicated in cognitive processes such as feeding and defensive behaviors $[8,9]$. Although similar functions are expected in the brain of $O$. minor, the contribution of each subregion to the behavior of $O$. minor needs to be tested by additional experiments. The optic and olfactory lobes were present on each lateral side (Fig. 3B and Supplementary Materials 4). With statocysts, these periesophageal lobes seem to relay sensory information to other parts of the brain. Some of the known lobes in the brains of $O$. vulgaris were difficult to detect in $O$. minor, and thus additional staining studies will be needed to locate these. In addition, it is possible that some subregions that may be involved in the inhabitance of $O$. minor in mudflats are unique or different from those of the $O$. vulgaris, with respect to the relative volume and microcircuitry level. Further studies addressing this issue would be of great interest. 
A

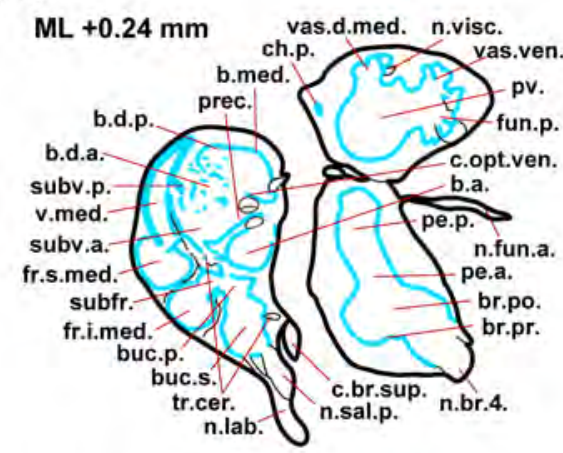

B
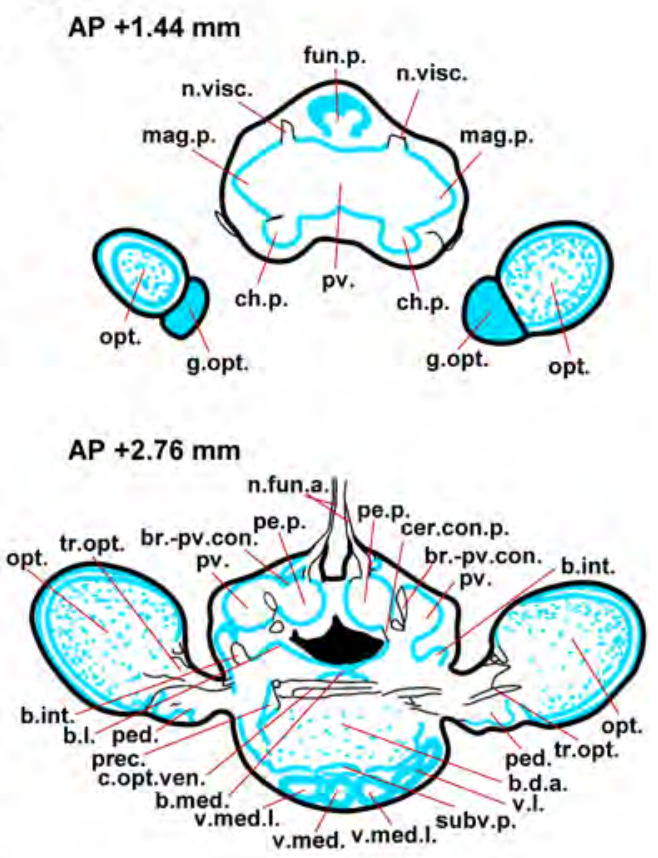

AP $+4.21 \mathrm{~mm}$

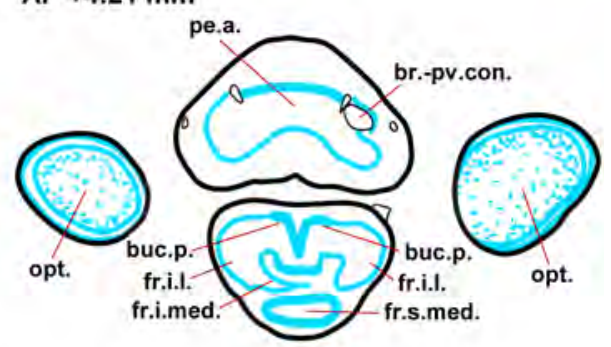

$A P+5.24 \mathrm{~mm}$

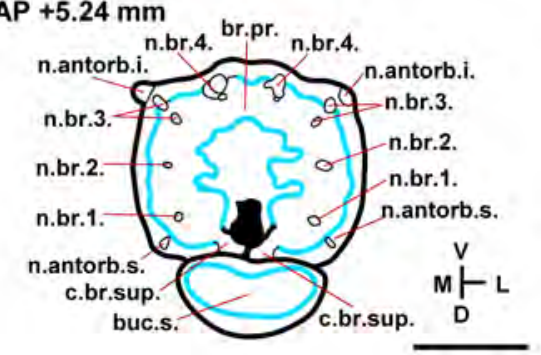

Fig. 3. Representative images of the delineations with their coordinates and identified subregions drawn on a selected sagittal (A) and coronal (B) brain section of Octopus minor. Scale bar=2 mm. AP, anterior-posterior axis; ML, medial-lateral axis; \pm , coordinate direction; A, anterior; P, posterior; D, dorsal; V, ventral; M, medial; L, lateral; b.a., anterior basal lobe; b.d.a., anterior dorsal basal lobe; b.d.p., posterior dorsal basal lobe; b.int., interbasal lobe; b.l., lateral basal lobe; b.med., median basal lobe; br.po., postbrachial lobe; br.pr., prebrachial lobe; br.-pv.con.,brachio-palliovisceral connective; buc.p., posterior buccal lobe; buc.s., superior buccal lobe; c.br.sup., suprabrachial commissure; c.opt.ven., ventral optic commissure; cer.-br.con., cerebro-brachial connective; cer.con.p, posterior cerebral connective; ch.p., posterior chromatophore lobe; fri.l., lateral inferior frontal lobe; fr.i.med., median inferior frontal lobe; fr.s.l., lateral superior frontal lobe; fr.s.med., median superior frontal lobe; fun.p., posterior funnel lobe; g.opt., optic gland; mag.d., dorsal magnocellular lobe; mag.p., posterior magnocellular lobe; n.antorb.s., superior antorbital nerve; n.antorb.i., inferior antorbital nerve; n.br.1., first brachial nerve; n.br.2., second brachial nerve; n.br.3., third brachial nerve; n.br.4., fourth brachial nerve; n.col., collar nerve; n.fun.a., anterior funnel nerve; n.fun.p., posterior funnel nerve; n.lab., labial nerve; n.pal., pallial nerve; n.sal.p., posterior salivary nerve; n.st., static nerve; n.visc., visceral nerve; opt., optic lobe; pe.a., anterior pedal lobe; pe.p., posterior pedal lobe; ped., peduncle lobe; ped.sub., subpedunculate lobe; prec., precommissural lobe; pv., palliovisceral lobe; subfr., subfrontal lobe; subv.a., anterior subvertical lobe; subv.p., posterior subvertical lobe; tr.br.-opt., brachial-optic lobe tract; tr.cer., cerebral tract; tr.opt., optic tract; v.l., lateral vertical lobe gyrus; v.med., median vertical lobe gyrus; v.med.l., medio-lateral vertical lobe gyrus; vas.d.l., lateral dorsal vasomotor lobe; vas.d.med., median dorsal vasomotor lobe; vas.ven., ventral vasomotor lobe. 


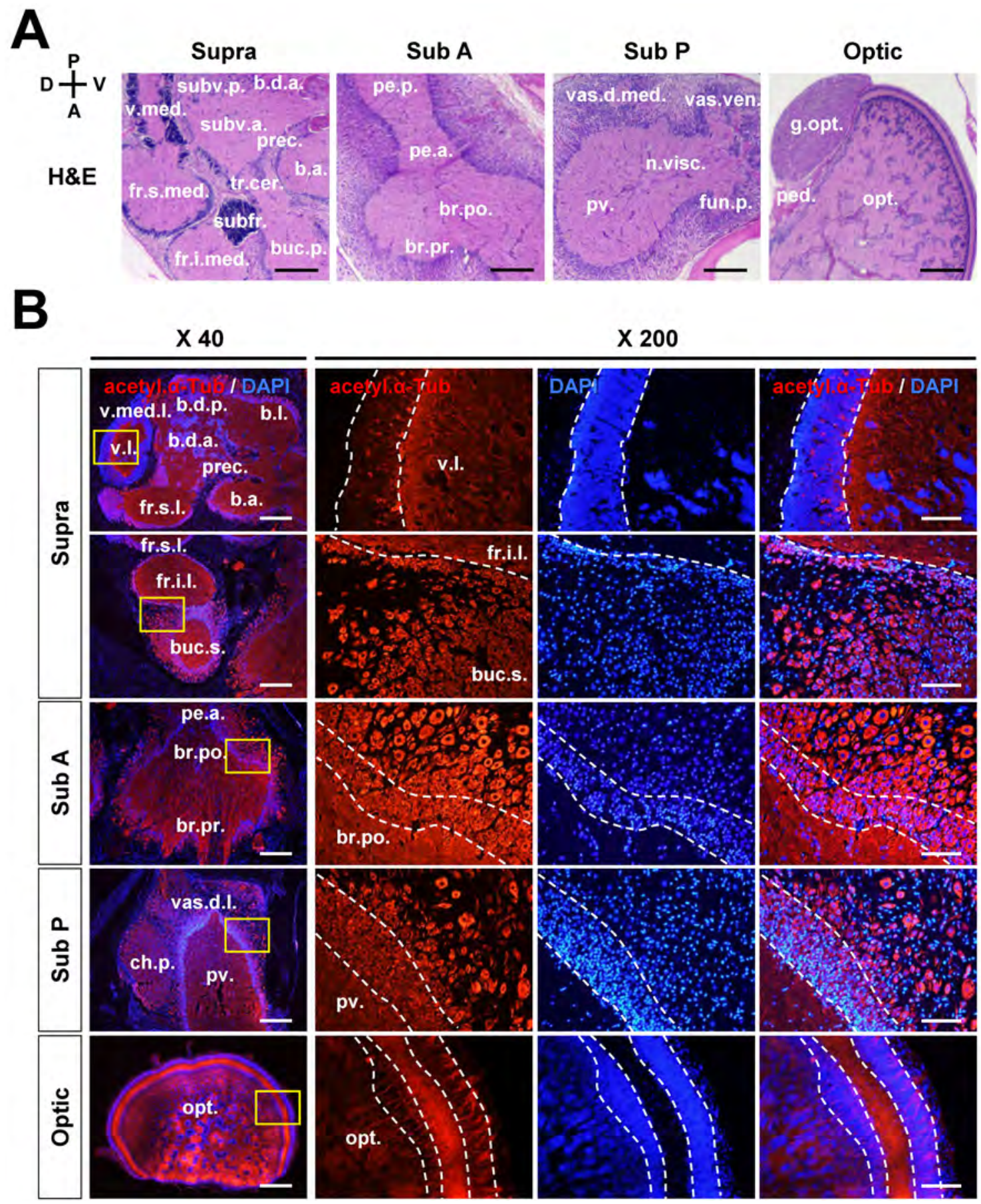

Fig. 4. Detailed view of sagittal sections of the brain of Octopus minor, shown according to the gross anatomy. (A) Representative magnified images at approximately $+0.36 \mathrm{~mm}$ from the midline and visualized by Hematoxylin and Eosin staining. Scale bar $=500 \mu \mathrm{m}$. (B) Representative magnified images at approximately $+1.62 \mathrm{~mm}$ from the midline and visualized by anti-acetylated $\alpha$-tubulin antibody and DAPI staining. Scale bar at $\times 40=400 \mu \mathrm{m}$ and at $\times 200=100 \mu \mathrm{m}$. H\&E, Hematoxylin and Eosin staining; acetyl. $\alpha$-Tub, acetylated $\alpha$-tubulin; DAPI, 4',6-diamidino-2-phenylindole; Supra, supraesophageal mass; Sub A, anterior part of the subesophageal mass; Sub P, posterior part of the subesophageal mass; Optic, optic lobe; A, anterior; P, posterior; D, dorsal; V, ventral; b.a., anterior basal lobe; b.d.a., anterior dorsal basal lobe; b.d.p., posterior dorsal basal lobe; b.l., lateral basal lobe; br.po., postbrachial lobe; br.pr., prebrachial lobe; buc.p., posterior buccal lobe; buc.s., superior buccal lobe; ch.p., posterior chromatophore lobe; fri.l., lateral inferior frontal lobe; fr.i.med., median inferior frontal lobe; fr.s.l., lateral superior frontal lobe; fr.s.med., median superior frontal lobe; fun.p., posterior funnel lobe; g.opt., optic gland; n.visc., visceral nerve; opt., optic lobe; pe.a., anterior pedal lobe; pe.p., posterior pedal lobe; ped., peduncle lobe; prec., precommissural lobe; pv., palliovisceral lobe; subfr., subfrontal lobe; subv.a., anterior subvertical lobe; subv.p., posterior subvertical lobe; tr.cer., cerebral tract; v.l., lateral vertical lobe gyrus; v.med., median vertical lobe gyrus; v.med.l., medio-lateral vertical lobe gyrus; vas.d.l., lateral dorsal vasomotor lobe; vas.d.med., median dorsal vasomotor lobe; vas.ven., ventral vasomotor lobe. 


\section{Heterogeneity of cell-types in the dense cell layers}

From the H\&E images of the supraesophageal and subesophageal mass, we found that the thickness and cell density of Hematoxylin-positive layers markedly varied depending upon the region (Fig. 4A). The optic lobe showed a distinctive structure, comprising islands of cells and a thin double Hematoxylin-positive layer near the surface (Fig. 4A). In the magnified images, the size of cellular nuclei in Hematoxylin-positive layer seemed to be diverse (image not shown). Therefore, we questioned the cellular identity of the dense Hematoxylin-positive cell layers of the O. minor brain, and sought to determine whether these layers contain neurons, as in the mammalian hippocampal formation [18], or non-neuronal cells, as in the Bergmann glial cell layer of the mammalian cerebellum [19]. To address this issue, we performed immunostaining using an anti-acetylated $\alpha$-tubulin antibody, which was previously utilized for visualizing neuronal somata and axons in many other cephalopod species [20-24], followed by counterstaining with DAPI. The somata of nerve cells showed a strong immunoreactivity with DAPI staining, whereas immunoreactivity without DAPI staining was observed in the regions with neuronal fibers. We found that the proportion of neurons in the DAPIpositive cell layers, the neuronal distribution patterns, and the size of neuronal somata varied markedly depending upon the region (Fig. 4B). Compared with the lobes of the subesophageal mass, those of the supraesophageal mass showed a considerably more diverse staining pattern. Interestingly, most of the cells in the thick dense DAPI-positive cell layer of the vertical lobe (v.l.), which is also known to function in the learning and memory process in $O$. vulgaris [8], were found to be non-neuronal cells (Fig. 4B). The subesophageal mass and optic lobe (opt.) showed intrinsic neuronal distribution patterns with less diversity (Fig. 4B). These results indicate the heterogeneity of neuronal subtypes as well as the presence of diverse and complex neural circuits in each brain subregion. Additional staining studies to identify subtypes of neurons and non-neuronal cells, possibly glia, and determination of the physiological functions of non-neuronal cell layers will provide interesting areas of focus in future research.

Although individual differences in brain size according to body weight and tissue shrinkage after formalin fixation are factors that should be taken into consideration, this study, nevertheless, represents an initial step towards characterizing the brain of $O$. minor. To the best of our knowledge, this is the first brain atlas of an Octopodiformes species as well as that of an adult cephalopod. Similar to the brain atlases of other animals, this atlas will enable site-specific approaches to brain stimulation, gene knockdown, and gene expression analyses in the O. minor brain. Although our findings have raised many questions regarding the cellular identity of neurons and non-neuronal cells for further investigation, we anticipate that the $O$. minor brain atlas compiled in the present study will make a valuable contribution to comparative neuroscience research.

\section{ACKNOWLEDGEMENTS}

This work was supported by the Research Program (2018M00900) of the National Marine Biodiversity Institute of Korea (MABIK) funded by the Ministry of Oceans and Fisheries, Korea. We thank Dr. Hye Suck An for foundation and stimulation of Octopus minor research in MABIK. We also thank director Hwang Bok Lee, Dr. Chun Cheol Kim, Mr. Ju Jeong, Dr. Hyun Ho Jeong and Dr. Yun Seol Kim (Oceans \& Fisheries Science Institute Western Departments) for maintaining the animals.

\section{REFERENCES}

1. Young RE, Vecchione M, Mangold KM (2018) Cephalopoda Cuvier 1797. Octopods, Squids, Nautiluses, etc. in the Tree of Life Web Project [Internet]. [cited 2018 Jul 13; updated 2018 Feb 20]. Available from: http://tolweb.org/Cephalopoda.

2. Hochner B, Shomrat T, Fiorito G (2006) The octopus: a model for a comparative analysis of the evolution of learning and memory mechanisms. Biol Bull 210:308-317.

3. Finn JK, Tregenza T, Norman MD (2009) Defensive tool use in a coconut-carrying octopus. Curr Biol 19:R1069-R1070.

4. Yamamoto M, Shimazaki Y, Shigeno S (2003) Atlas of the embryonic brain in the pygmy squid, Idiosepius paradoxus. Zool Sci 20:163-179.

5. Koizumi M, Shigeno S, Mizunami M, Tanaka NK (2016) Three-dimensional brain atlas of pygmy squid, Idiosepius paradoxus, revealing the largest relative vertical lobe system volume among the cephalopods. J Comp Neurol 524:21422157.

6. Shigeno S, Kidokoro H, Tsuchiya K, Segawa S, Yamamoto M (2001) Development of the brain in the oegopsid squid, Todarodes pacificus: an atlas up to the hatching stage. Zool Sci 18:527-541.

7. Shigeno S, Kidokoro H, Tsuchiya K, Segawa S, Yamamoto M (2001) Development of the brain in the oegopsid squid, Todarodes pacificus: an atlas from hatchling to juvenile. Zool Sci 18:1081-1096.

8. Nixon M, Young JZ (2003) The brains and lives of cephalopods. Oxford University Press, Oxford.

9. Young JZ (1971) The anatomy of the nervous system of octopus vulgaris. Oxford University Press, Oxford. 
10. Voss NA, Sweeney M (1998) Systematics and biogeography of cephalopods. Volume II. Smithsonian Institution Press, Washington, D.C.

11. National Fisheries Research \& Development Institute (2006) Commercial cephalopods of the world-an illustrated guide. Yeamoonsa, Busan.

12. Jereb P, Roper C, Norman M, Finn J (2016) Cephalopods of the world. An annotated and illustrated catalogue of cephalopod species known to date. Vol. 3. Octopods and vampire squids. Food and Agriculture Organization of the United $\mathrm{Na}$ tions, Rome.

13. Cheng R, Zheng X, Lin X, Yang J, Li Q (2012) Determination of the complete mitochondrial DNA sequence of Octopus minor. Mol Biol Rep 39:3461-3470.

14. Kang JH, Park JY, Choi TJ (2012) Genetic differentiation of octopuses from different habitats near the Korean peninsula and eastern China based on analysis of the mDNA cytochrome C oxidase 1 gene. Genet Mol Res 11:3988-3997.

15. Folmer O, Black M, Hoeh W, Lutz R, Vrijenhoek R (1994) DNA primers for amplification of mitochondrial cytochrome c oxidase subunit I from diverse metazoan invertebrates. Mol Mar Biol Biotechnol 3:294-299.

16. Paxinos G, Franklin KB (2004) The mouse brain in stereotaxic coordinates. Academic Press, Cambridge, MA.

17. Jung SH, Kim S, Chung AY, Kim HT, So JH, Ryu J, Park HC, Kim CH (2010) Visualization of myelination in GFP-transgenic zebrafish. Dev Dyn 239:592-597.

18. Jo S, Yarishkin O, Hwang YJ, Chun YE, Park M, Woo DH,
Bae JY, Kim T, Lee J, Chun H, Park HJ, Lee DY, Hong J, Kim HY, Oh SI, Park SJ, Lee H, Yoon BE, Kim Y, Jeong Y, Shim I, Bae YC, Cho J, Kowall NW, Ryu H, Hwang E, Kim D, Lee CJ (2014) GABA from reactive astrocytes impairs memory in mouse models of Alzheimer's disease. Nat Med 20:886-896.

19. Yoon BE, Jo S, Woo J, Lee JH, Kim T, Kim D, Lee CJ (2011) The amount of astrocytic GABA positively correlates with the degree of tonic inhibition in hippocampal CA1 and cerebellum. Mol Brain 4:42.

20. Shigeno S, Yamamoto M (2002) Organization of the nervous system in the pygmy cuttlefish, Idiosepius paradoxus ortmann (Idiosepiidae, Cephalopoda). J Morphol 254:65-80.

21. Kingston AC, Wardill TJ, Hanlon RT, Cronin TW (2015) An unexpected diversity of photoreceptor classes in the longfin squid, Doryteuthis pealeii. PLoS One 10:e0135381.

22. Wollesen T, Sukhsangchan C, Seixas P, Nabhitabhata J, Wanninger A (2012) Analysis of neurotransmitter distribution in brain development of benthic and pelagic octopod cephalopods. J Morphol 273:776-790.

23. von Byern J, Wani R, Schwaha T, Grunwald I, Cyran N (2012) Old and sticky-adhesive mechanisms in the living fossil Nautilus pompilius (Mollusca, Cephalopoda). Zoology (Jena) 115:1-11.

24. Wollesen T, Loesel R, Wanninger A (2009) Pygmy squids and giant brains: mapping the complex cephalopod CNS by phalloidin staining of vibratome sections and whole-mount preparations. J Neurosci Methods 179:63-67. 